J. Clin. Chem. Clin. Biochem.

Vol. 23, 1985, pp. $231-240$

\title{
Beobachtungen über Fibrinogen als Störquelle bei heterogenen Enzym-Immunoassays
}

\author{
Von R. Allner \\ Zentrallabor der Städtischen Kliniken Fulda, Lehrkrankenhaus der Philipps-Universität Marburg
}

(Eingegangen am 31. März/10. September/30. November 1984)

\begin{abstract}
Zusammenfassung: Heterogene enzymimmunologische invitro-Teste werden durch Fibrinogen gestört. Die Auswirkungen auf das Testergebnis sind unterschiedlich und vom Testprinzip (kompetitive Bindungsanalyse oder Sandwich-Technik) abhängig. Es wird mit den Methoden zum Nachweis von Digoxin, Cortisol, $\alpha_{1-}$ Fetoprotein und der sauren Prostata-Phosphatase gezeigt, daß bei Einsatz von Plasma als Probematerial die Testverfahren in unterschiedlichem Ausmaß störanfällig werden können. An Hand der Digoxinbestimmung mit dem Enzymun-Test ${ }^{\circledR}$ Digoxin werden Möglichkeiten erörtert, den störenden Fibrinogeneffekt zu beseitigen. Nach thermischer Koagulation (Erhitzen des Plasmas auf $56^{\circ} \mathrm{C} 30$ Minuten lang) läßt sich Fibrinogen abtrennen, durch Harnstoffzusatz zum Reaktionsmilieu der Antigen-Antikörper-Reaktion kann die durch Fibrinogen bedingte Störung des Testes beeinflußt werden. Es ergeben sich Hinweise, daß die Harnstoffzusätze und die Inkubationsdauer jeweils dem Probenmaterial angepaßt werden müßten.
\end{abstract}

\section{Fibrinogen as a source of interference in heterogenous enzyme-immunoassays}

Summary: Fibrinogen interferes in heterogeneous enzyme immunological in vitro tests. The effect on the test result is variable and depends on the test principle (competitive binding analysis or sandwich technique). In the tests for digoxin, cortisol, $\alpha_{1}$-fetoprotein and acid prostate phosphatase, the use of plasma as sample material resulted in varying degrees of interference. In the case of the digoxin determination, using the Enzymun-Test ${ }^{\oplus}$ Digoxin, it is possible to abolish interference by fibrinogen by thermal coagulation (plasma is heated at $56^{\circ} \mathrm{C}$ for $30 \mathrm{~min}$ ) and removal of the fibrinogen. Alternatively, addition of urea to the antigenantibody reaction mixture decreases the interference caused by fibrinogen. The added quantity of urea and the time of incubation must be optimized for each sample material.

\section{Einführựng}

Hersteller von enzymimmunologischen in vitro-Tests geben in der Regel an, welche Probenmaterialien in ihrem Test zur Analyse eingesetzt werden können. Im allgemeinen wird Serum empföhlen. Die Möglichkeit, Serum als auch Plasma einsetzen zu können, erscheint sinnvoll, da es hierzu im klinischen Laboratorium gute Gründe gibt (1). Aus eigenen Erfahrungen soll in dieser Arbeit darauf hingewiesen werden, daß die erreichten Ergebnisse aus Serum und Plasma aber weit voneinander abweichen können. Fibrinogen wird in diesen Zusammenhängen als Störquelle angesehen, dessen störender Einfluß offenbar nicht immer in ausreichendem $\mathrm{Ma} ß$ in dem zur Verfügung stehenden Testverfahren ausgeschaltet werden kann. Die Abweichungen der erreichten Testergebnisse sind in ihren Ausmaßen geeignet, zu einer Fehleinschätzung der klinischen Situation des Patienten zu führen. Im Rahmen eigener Untersuchungen ergaben sich keine Hinweise darauf, die Antikoagulantien selbst als mögliche Störquellen anzusehen. Überraschend ist, daß auch solche Teste, die ausdrücklich vom Hersteller zum Einsatz von Serum als auch Plasma angeboten werden, dennoch erhebliche Beeinflussungen der Testergebnisse durch das Probenmaterial zeigen. In der Literatur (2) finden sich bis jetzt wenige Hinweise zu 
diesem Problemkreis. Mit geeigneten Zusätzen im entscheidenden Reaktionsschritt der Testverfahren besteht wohl eine Möglichkeit, der Störung des Fibrinogens entgegenzuwirken. Offenbar sind die zu dieser Problemlösung einsetzbaren Möglichkeiten aber nicht immer in der Lage, die ganze quantitative Variationsbreite der Störquelle Fibrinogen vollständig zu erfassen.

\section{Material und Methoden}

Benutzt wurden folgende enzymimmunologische in vitro-Teste, um den störenden Einfluß von Fibrinogen im Plasma auf das Testergebnis zu untersuchen:

$\alpha_{1}$-Fetoprotein (AFP) mit dem Enzymun-Testiti AFP aus dem Programm Enzymdiagnostica der Firma Boehringer, Mannheim, Best.-Nr.: 197386,

Cortisol mit dem Test Cortisol-sopheia ${ }^{\text {TM }}$ aus dem Programm der Firma Diagnostica Products Corporation 5700 West $96^{\text {th }}$ Street, Los Angeles. California 90045; Vertrieb in Deutschland durch Hermann. Biermann. Medizinische Systeme GmbH. Lindenstr. 16, 6345 Bad Nauheim, Best.-Nr. SKC O1,

Digoxin (einschließlich $\beta$-Methyldigoxin, $\alpha$-Acetyldigoxin, $\beta$ Acetyldigoxin) mit dem Enzymun-Test ${ }^{10}$ Digoxin aus dem Testprogramm Immundiagnostica der Firma Boehringer, Mannheim, Best.-Nr. 199656

Saure Prostata-Phosphatase (PAP), mit dem Test Enzygnost PAP, aus dem Programm der Behringwerke AG, Marburg, Best.-Nr. $\emptyset$ SSP 10 ,

Folgende zusätzliche Lösungen und Reagenzien wurden benutzt:

Human-Albumin-Lösung $50 \mathrm{~g} / \mathrm{l}$ aus dem Programm des Herstellers Deutsches Rotes Kreuz Hessen,

Humane gereinigte Fibrinogenfraktion der Firma Immuno GmbH Heidelberg,

Harnstoff, zur Analyse aus dem Programm der Firma E. Merck, Darmstadt, Best.-Nr. 8487,

Digoxin, Rcinsubstanz aus dem Programm der Firma E. Merck, Darmstadt, Best.-Nr. 3054,

Zusätzlich benutzte Untersuchungsverfahren:

Fibrinogen wurde nach der Methode von Clauss (3) bestimmt unter Verwendung einer Testkombination der Firma Boehringer, Mannheim, aus dem Programm Gerinnungsdiagnostica, Best.-Nr. 126691.

Zur Eiweißbestimmung wurde die Biuret-Methode nach T.E. Weichselbaum (4) benutzt.

Die Ermittlung der Ergebnisse erfolgte bei den enzymimmunologischen Tests über eine manuell gezeichnete Bezugskurve, die aus den Mittelwerten der Absorbanz mitgeführter Standards erstellt wurde. Alle aufgeführten Einzelwerte ergeben sich aus Dreifachmessungen. Die Pipettierungen wurden manuell vorgenommen. Die Messungen erfolgten am Digitalphotometer mit Drucker PCP 6121 der Firma Eppendorf Gerätebau GmbH, Hamburg.

Die untersuchten Seren und Plasmen stammten von Patienten der Städt. Kliniken in Fulda. Die Blutentnahmen erfolgten stets morgens am nüchternen Patienten. Blut wurde aus der gestauten Cubitalvene entnommen. Zur Plasmagewinnung wurden folgende Antikoagulantien eingesetzt:
1. $38 \mathrm{~g} / \mathrm{l}$ Natrium-Citrat-Lösung: das Mischungsverhältnis betrug 9 Teile venöses Blut und ein Teil Natrium-Citrat-Lösung.

2. Kalium-EDTA (Ethylendiamin-tetraessigsäure, Di-Kaliumsalz, Dihydrat): zu $3 \mathrm{mg}$ Kalium-EDTA wurden $4 \mathrm{ml}$ venöses Vollblut gegeben, eingesetzt wurden entsprechend präparierte Röhrchen der Firma Sarstedt, Köln, Best.-Nr.: 05277. .

3. Kalium-Heparinat: zu 0,6 mg Ammonium-Heparinat kamen $4 \mathrm{ml}$ venöses Blut. Eingesetzt wurden entsprechend präparierte Röhrchen der Firma Sarstedt Köln, Best.-Nr.: 02.264.

Die Qualitätssicherung für alle durchgeführten Tests erfolgte durch tägliche Koñtrollen von Präzision und Richtigkeit an Hand der vom Hersteller mitgelieferten Kontrollseren.

\section{Statistische Methoden}

Zur Charakterisierung der Wiederholbarkeit in der Serie wurde das arithmetische Mittel $(\overline{\mathbf{x}})$, die Standard-Abweichung $(\sigma)$ und der Variations-Koeffizient (VK) bei Mehrfach-Bestimmungen ermittelt. Die Auswertung der Ergebnisse der im Sinne von Paar-Analysen angelegten Versuchsanordnung erfolgte durch die Berechnung des Korrelations-Koeffizienten ( $r$ ), der Regressionsgeraden, charakterisiert für $y=a_{0}+a_{1} \cdot x$, wobei $a_{0}$ der Schnittpunkt der Geraden mit der $y$-Achse und $a_{1}$ die Steigung der Geraden ist und für $x=b_{0}+b_{1} \cdot y$, wobei $b_{0}$ der Schnittpunkt der Geraden mit der $\mathrm{x}$-Achse und $\mathrm{b}_{1}$ die Steigung mit der Geraden bedeutet, der Anwendung des t-Test bei Korrelationen, sowie durch den t-Test für Paar-Analysen nach Student. Die Signifikanz der Unterschiede wird in Wahrscheinlichkeitsgeraden $p$ angegeben.

\section{Ergebnisse}

\section{Qualitätskontrolle}

Tabelle 1 enthält die Ergebnisse der Qualitätssicherung für die durchgeführten enzymimmunologischen Teste in mehreren Konzentrationsbereichen in der

Tab. 1. Präzision in der Serie.

$\begin{array}{llll}\overline{\mathrm{x}} & \sigma & \mathrm{VK} & \mathrm{N} \\ & & \%)\end{array}$

\begin{tabular}{|c|c|c|c|c|}
\hline \multirow[b]{2}{*}{$\begin{array}{l}\text { Enzymun-Test Digoxin } \\
\text { Digoxin }\end{array}$} & \multicolumn{2}{|c|}{$(\mathrm{nmol} / \mathrm{l})$} & \multirow[b]{2}{*}{$\begin{array}{l}9,4 \\
9,1\end{array}$} & \multirow[b]{2}{*}{$\begin{array}{l}12 \\
12\end{array}$} \\
\hline & $\begin{array}{l}1,81 \\
2,32\end{array}$ & $\begin{array}{l}0,17 \\
0,21\end{array}$ & & \\
\hline & \multicolumn{2}{|c|}{$(\mu \mathrm{g} / \mathrm{l})$} & & \\
\hline \multirow[t]{2}{*}{$\begin{array}{l}\text { Enzymun-Test }{ }^{\oplus} \text { AFP } \\
\alpha_{1} \text {-Fetoprotein }\end{array}$} & $\begin{array}{r}3,5 \\
61,4 \\
131,0\end{array}$ & $\begin{array}{l}0,28 \\
0,86 \\
1,96\end{array}$ & $\begin{array}{l}8,2 \\
1,4 \\
1,5\end{array}$ & $\begin{array}{l}10 \\
10 \\
10\end{array}$ \\
\hline & \multicolumn{2}{|c|}{$(\mu \mathrm{g} / \mathrm{l})$} & & \\
\hline \multirow[t]{3}{*}{$\begin{array}{l}\text { Enzygnost }^{\circledR} \text {-PAP } \\
\text { saure Prostata- } \\
\text { Phosphatase }\end{array}$} & $\begin{array}{c}0,66 \\
1,05 \\
26,0\end{array}$ & $\begin{array}{l}0,11 \\
0,09 \\
1,95\end{array}$ & $\begin{array}{r}17,0 \\
8,3 \\
7,5\end{array}$ & $\begin{array}{l}20 \\
10 \\
10\end{array}$ \\
\hline & \multicolumn{2}{|c|}{$(\mathrm{nmol} / \mathrm{l})$} & & \\
\hline & $\begin{array}{r}67,3 \\
224,1 \\
678,9\end{array}$ & $\begin{array}{c}4,9 \\
12,53 \\
28,21\end{array}$ & $\begin{array}{l}7,2 \\
5,6 \\
4,2\end{array}$ & $\begin{array}{l}10 \\
10 \\
10\end{array}$ \\
\hline
\end{tabular}


Serie. Zum Einsatz kamen Poolseren. Auf den Hinweis der Präzision von Tag zu Tag wird in dieser Arbeit verzichtet, da die Untersuchungen im Rahmen der Fragestellung in serieller Folge durchgeführt wurden.

Ergebnisvergleiche aus Serum- und Plasmaproben

$\alpha_{1}$-Fetoprotein (AFP) nach dem Enzymun-Test ${ }^{\circledR}$ AFP bestimmt, zeigt in geringem Maße Abweichungen der gefundenen Wertepaare zwischen Serum und Plasma (Abb. 1). Der Korrelations-Koeffizient aus den Wertepaaren drückt einen stark positiven Zusammenhang aus. Die Ergebnisse aus beiden verschiedenen Probenmaterialien sind dennoch unterschiedlich, gesichert im t-Test für Paar-Analysen mit einem $\mathrm{p}<0,001$. Im Plasma werden demnach etwas niedere Werte gegenüber dem Serum gefunden.

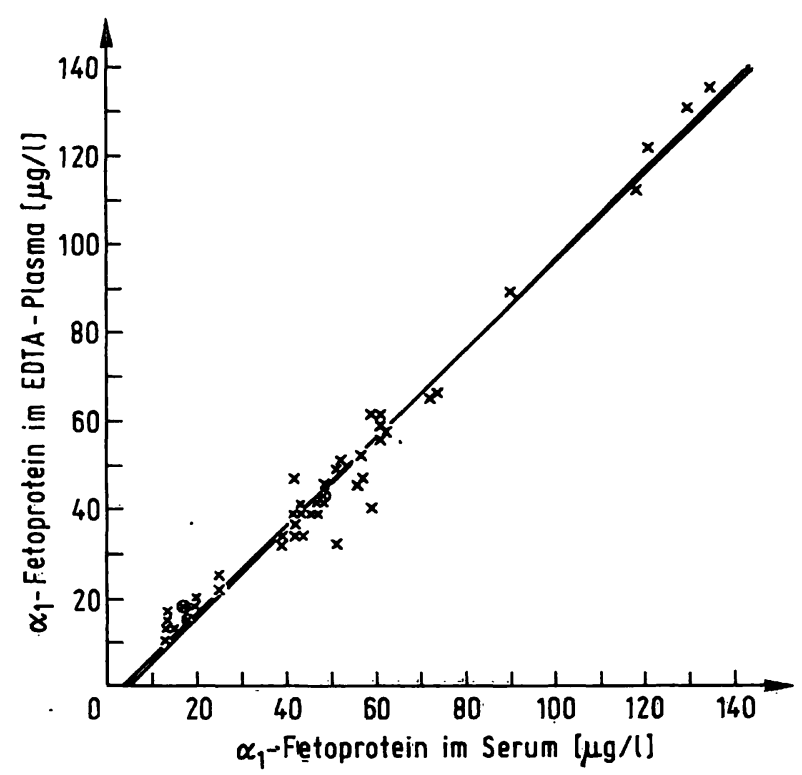

Abb. 1. Korrelation zwischen Werten für $\alpha_{1}$-Fetoprotein im Serum und im EDTA-Plasma:

$\mathrm{N} \equiv 48 ; \mathrm{x}=4,1356+0,9977 \cdot \mathrm{y}$;

$y=-3,1341+0,980 \cdot x ; \quad r=0,9888 ; t=44,9503$;

$\mathrm{p}<0,001$

$t$-Tèst für Paar-Analysen:

$t=6,6694 ; p<0,001$.

Aụch bei dẹr Beștịmmung des Cortisols mit dem Cortisol-sopheia ${ }^{\mathrm{TM}}$-Test stehen die Wertepaare aus den beiden Proben Plasma und Serum in einem positiven Zusammenhang. Unterschiede zwischen Serum und Plasma lassen sich statistisch nach dem t-Test für Paar-Analysen nicht sichern. Die Ergebnisse sind unabhängig von der Wahl des Antikoagulans (Abb. 2a, b, c).
Eindeutig gesichert nach dem t-Test für Paar-Analysen sind die Unterschiede der ermittelten Ergebnisse aus Serum- und Plasmaproben bei der Bestimmung des Digoxins nach dem Enzymun-Test ${ }^{\circledR}$ Digoxin. Zwischen den Wertepaaren ergeben sich nach dem Korrelations-Koeffizienten positive Zusammenhänge. Im Plasma werden stets höhere Werte als im Serum gefunden (2). Auch hier läßt sich keine Abhängigkeit vom eingesetzten Koagulans erkennen (Abb. $3 a, b$ ).

Bei der Bestimmung der sauren Prostata-Phosphatase (PAP) im Serum und Plasma nach der enzym-immunologischen Methode Enzygnost ${ }^{\circledR}$ PAP waren die gemessenen Werte im Plasma gegenüber dem Serum erniedrigt. Die Untersuchungen wurden an Plasmen durchgeführt, die einen Zusatz von Citrat oder EDTA enthielten. Die gefundenen Unterschiede sind nach dem $t$-Test für Paar-Analysen jeweils signifikant (Abb. 4a, b).

Die Erfahrungen zeigen, daß bei der Bestimmung der sauren Prostata-Phosphatase im Plasma im klinisch relevanten Entscheidungsbereich falsch normale Konzentrationen von saurer Prostata-Phosphatase gemessen werden können.

Im wesentlichen besteht der qualitative Unterschied zwischen Plasma und Serum im Fibrinogengehalt. Es liegt deshalb der Verdacht nahe, daß Fibrinogen im Immunoassay als Störquelle in Betracht kommt. Diese Fragestellung wurde an geeigneten Modellproben untersucht. Als Probenmaterial wurde eine Albuminlösung (Proteinkonzentration $50 \mathrm{~g} / \mathrm{l}$ ) verwendet. Durch Aufstockung dieser Lösung mit verschiedenen Fibrinogenmengen wurden Modellproben hergestellt. Die Reinheit der Proteine wurde mit der Trägerelektrophorese überprüft. Danach war die Proteinmatrix der Modellproben frei von Immunglobulinen. Die eingewogenen Fibrinogenmengen in den Modellproben wurden mit der Methode nach Clauss (3) nachbestimmt. Die quantitativen Angaben über die Fibrinogenmengen in den Modellproben entsprechen somit gerinnungsphysiologisch wirksamen Substanzmengen.

Die Bestimmung von $\alpha_{1}$-Fetoprotein (AFP) erfolgte an Modellproben mit und ohne Zusatz von $\alpha_{1}$-Fetoprotein mit dem Enzymun-Test ${ }^{\circledR}$ AFP. Die eingesetzten Mengen von $\alpha_{1}$-Fetoprotein waren in allen Modellproben gleich. Die Wiederfindung der zugesetzten Mengen an $\alpha_{1}$-Fetoprotein nimmt mit steigenden Fibrinogenmengen ab. In den Modellproben ohne Aufstockung mit $\alpha_{1}$-Fetoprotein haben steigende Fibrinogenmengen keinen Einfluß auf die Meßergebnisse (Abb. 5): 


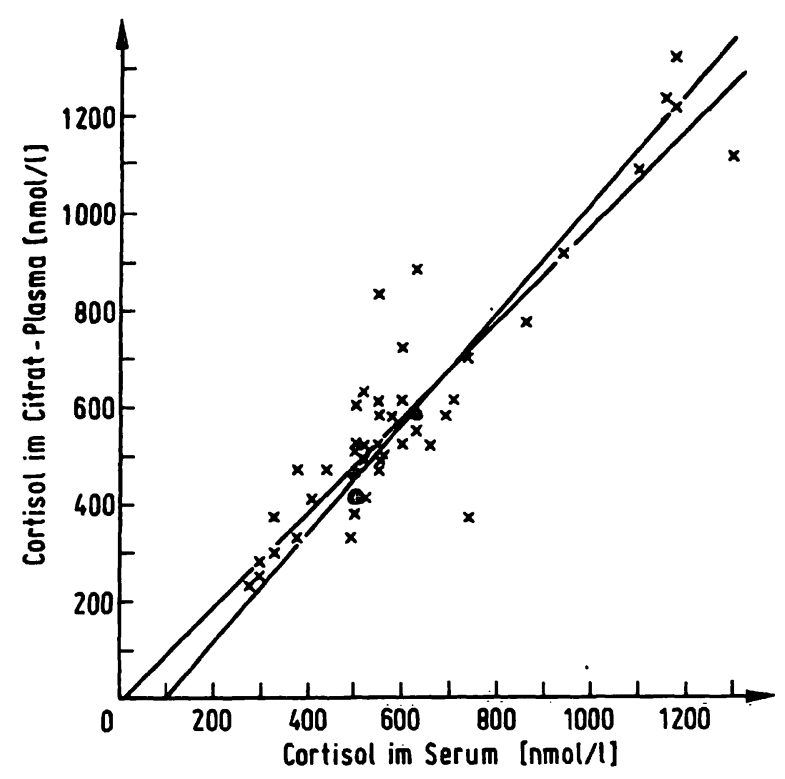

a) Citrat-Plașma

$N=52 ; x=100,2+0,88 \cdot y ; y=10,03+0,97 \cdot x ;$ $r=0,9236 ; t=17,03 ; p<0,001$

t-Test für Paar-Analysen:

$\mathrm{t}=1,8447 ; 0,05<\mathrm{p}<0,1$

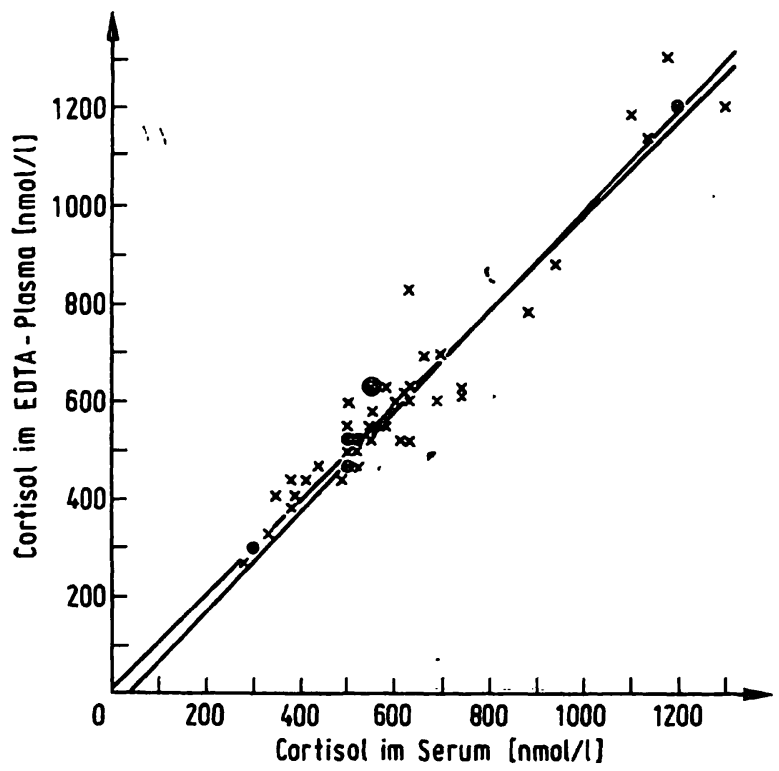

b) EDTA-Plasma

$\mathrm{N}=51 ; \mathrm{x}=25,68+0,96 \cdot \mathrm{y} ; \mathrm{y}=13,09+0,97 \cdot \mathrm{x}$ $r=0,9688 ; t=27,36 ; p<0,001$

t-Test für Paar-Analysen:

$t=0,4616 ; 0,6<p<0,7$

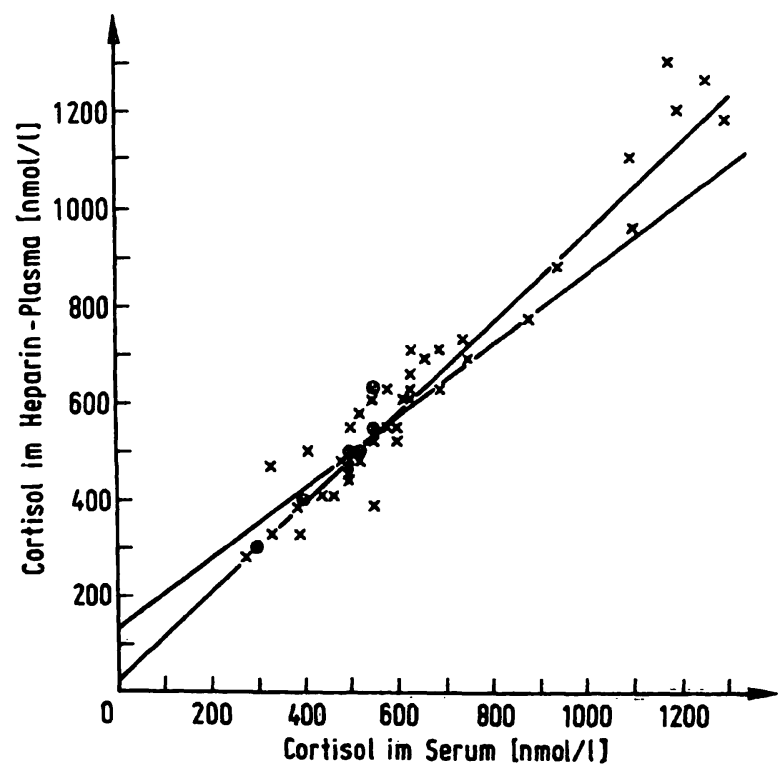

c) Heparin-Plasma

$N=52 ; x=29,64+1,07 \cdot y ; y=129,8+0,77 \cdot x ;$ $r=0,9095 ; t=15,47 ; p<0,001$

t-Test für Paar-Analysen:

$\mathrm{t}=0,2512 ; 0,7<\mathrm{p}<0,8$

Abb. 2. Korrelation zwischen Cortisol-Werten im Serum und im Plasma.

J. Clin. Chem. Clin. Biochem. / Vol. 23, 1985 / No. 4 

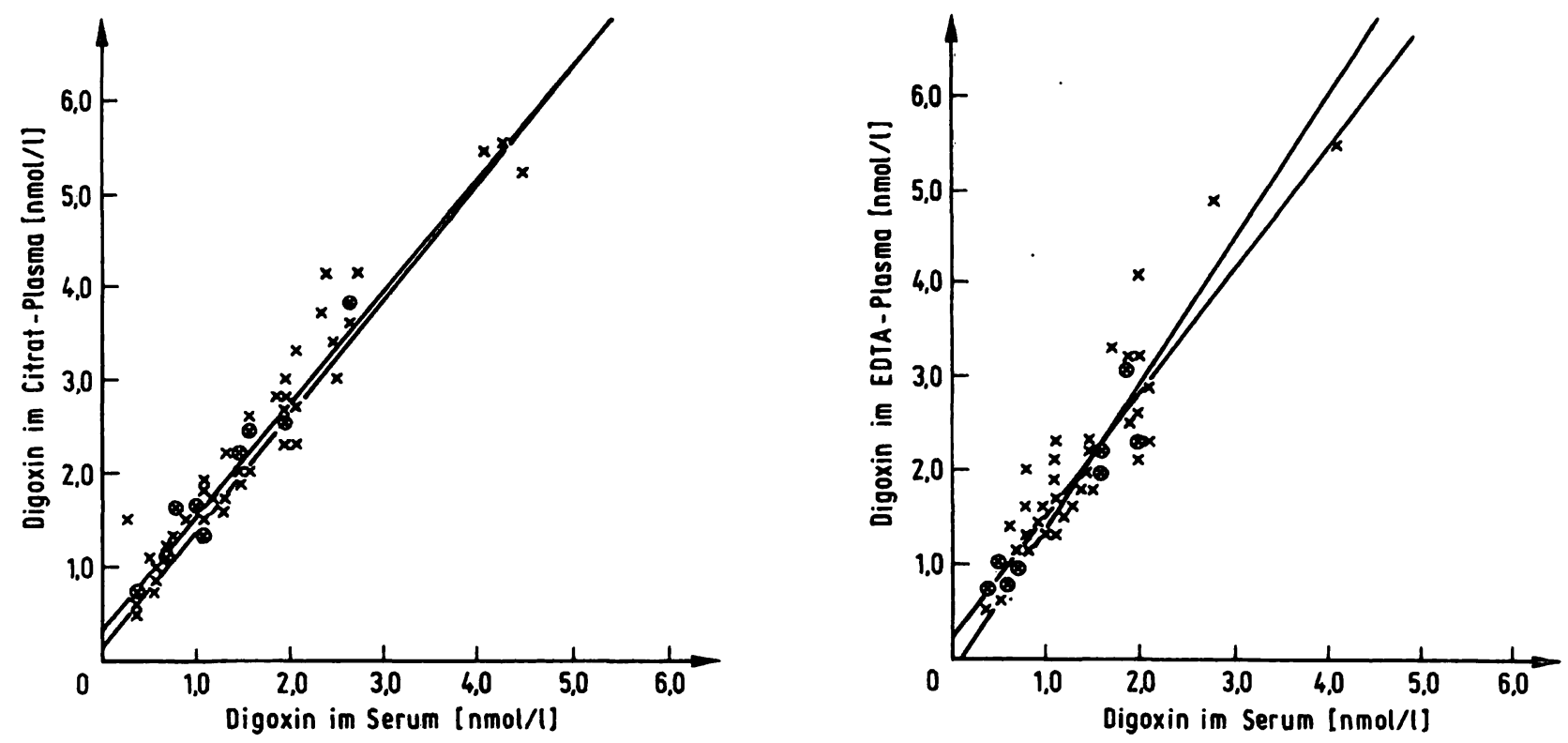

Abb. 3. Korrelation zwischen Digoxin-Werten im Serum und im Plasma:

a) Citrat-Plasma

$\mathrm{N}=58 ; \mathrm{x}=-0,132+0,76 \cdot \mathrm{y} ; \mathrm{y}=0,328+1,21 \cdot \mathrm{x}$ $r=0,9594 ; t=25,92 ; p<0,001$

$\mathrm{t}$-Test für Paar-Analysen:

$t=-13,58 ; p<0,001$ b) EDTA-Plasma

$N=57 ; x=0,093+0,64 \cdot y ; y=0,231+1,29 \cdot x ;$ $r=0,9047 ; t=15,60 ; p<0,001$

t-Test für Raar-Analysen:

$t=9,25 ; p<0,001$
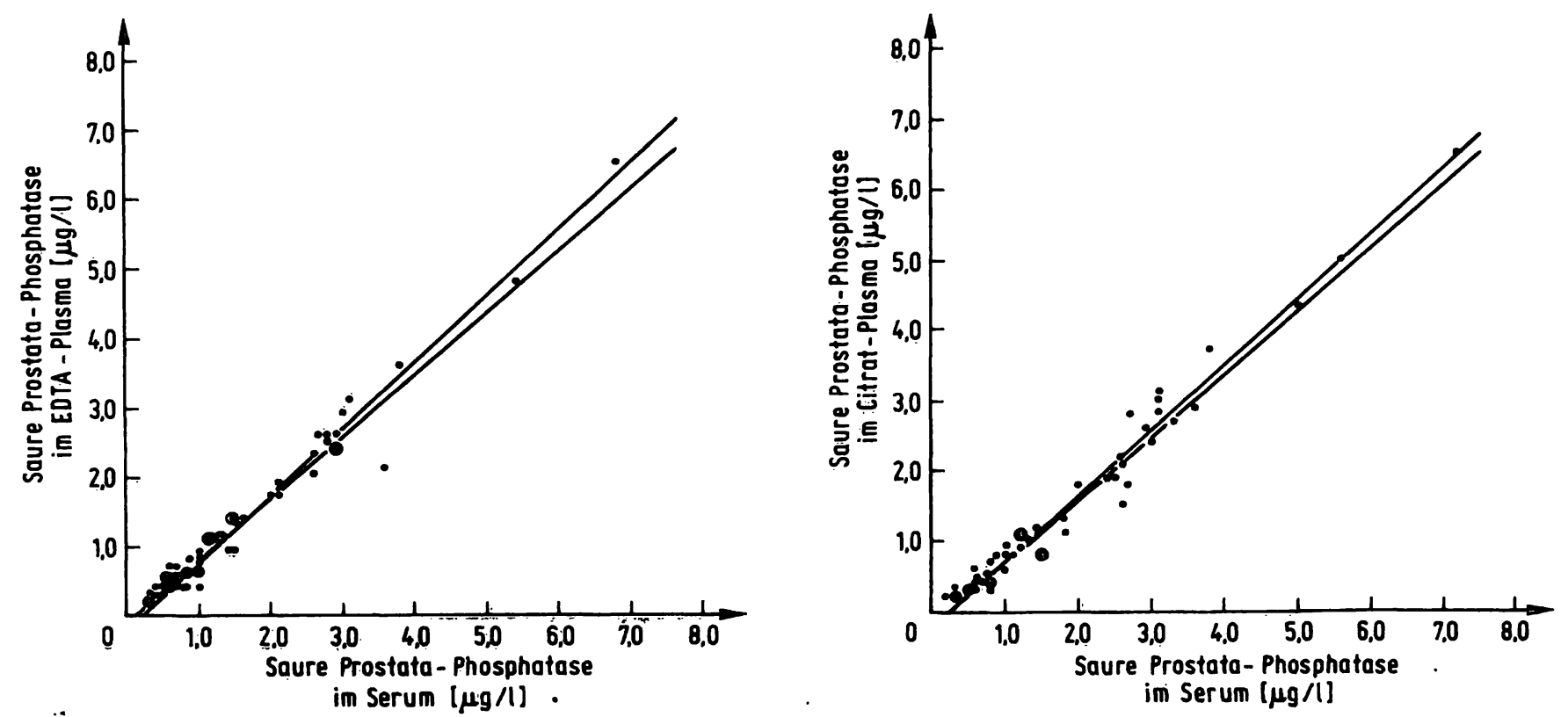

Abb. 4. Korrelation zwischen Werten für saure Prostata-Phosphatase im Serum und im Plasma:

a) EDTA-Plasma

$\mathrm{N}=50 ; \mathrm{x}=0,218+1,04 \cdot \mathrm{y} ; \mathrm{y}=-0,135+0,91 \cdot \mathrm{x}$

$r=0,9743 ; t=29,98 ; p<0,001$

t-Test für Paar-Analysen:

$t=6,93 ; p<0,001$ b) Citrat-Plasma

$N=50 ; x=0,236+1,08 \cdot y ; y=-0,155+0,89 \cdot x$ $r=0,9788 ; t=33,10 ; p<0,001$

t-Test für Paar-Analysen:

$\mathrm{t}=9,17 ; \mathrm{p}<0,001$ 


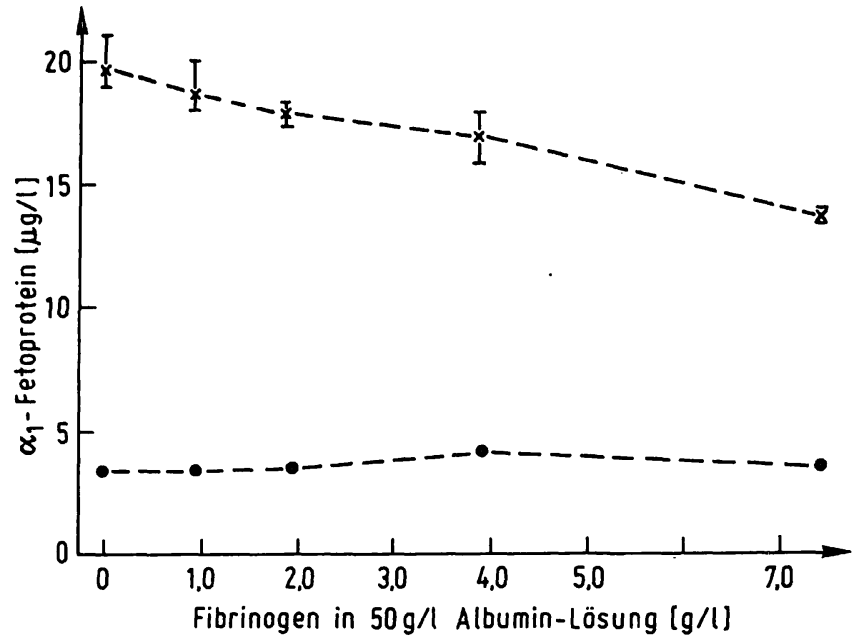

Abb. 5. Vortäuschung einer Abnahme von $\alpha_{1}$-Fetoprotein in einer Albuminlösung (Proteinkonzentration von 50 $\mathrm{g} / \mathrm{l})$ und einer zugesetzten Menge von $19 \mathrm{mg} / \mathrm{l} \alpha_{1}$-Fetoprotein in Abhängigkeit steigender Mengen Fibrinogen $(x-x)$. Keine Beeinflussung der Testergebnisse bei gleicher Probenzusammensetzung aber ohne Zusatz von $\alpha_{1}$-Fetoprotein $(\bullet--\rightarrow)$.

Im cortisolfreien Modellprobenmaterial werden bei der Cortisolbestimmung mit dem Cortisol-sopheia ${ }^{\mathrm{TM}}$ in Abhängigkeit der Fibrinogenmengen Cortisolmengen vorgetäuscht (Abb. 6).

Ähnliche Verhältnisse finden sich bei der Digoxinbestimmung nach dem Enzymun-Test ${ }^{\varpi 4}$ Digoxin. Mit steigenden Mengen Fibrinogen in den Modellproben werden entsprechende Digoxinmengen vorgetäuscht (Abb. 7).

Diese Beobachtungen lassen den Schluß zu, daß Fibrinogen in den untersuchten Modellproben als Störquelle in Betracht kommt. Zu ihrer Beseitigung bieten sich verschiedene Möglichkeiten an:

1. Recalcifizierung des Plasmas, Starten der Gerinnungskaskade, Abtrennung des Fibrins durch Zentrifugation.

2. Thermische Denaturierung des Fibrinogens und nachfolgende Abtrennung durch Zentrifugation (5), vorausgesetzt, daß die zu bestimmende Substanz thermostabil ist.

3. Zusatz geeigneter Stoffe zum Milieu der Immunreaktion mit dem Ziel, eine Störung durch Fibrinogen im Analysenverfahren zu vermeiden.

Durch eine geeignete Probenvorbehandlung wird eine bessere Vergleichbarkeit der Ergebnisse aus Plasma und Serum erreicht. Am Beispiel der Digoxinbestimmung mit dem Enzymun-Test ${ }^{\circledR}$ Digoxin können diese Möglichkeiten erörtert werden.

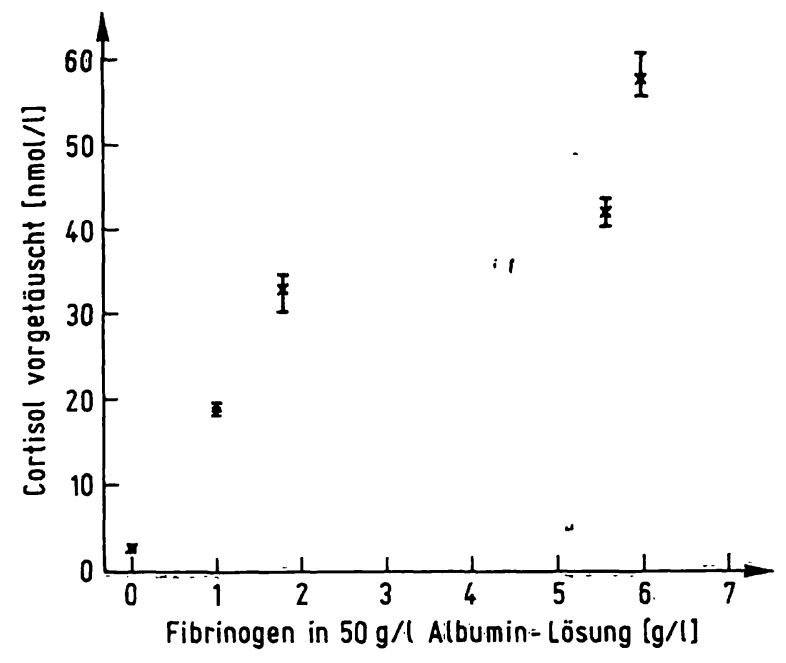

Abb. 6. Vortäuschung von Cortisol im cortisolfreien Milieu durch Fibrinogen; Matrix der Proben war eine Albuminlösung (Proteinkonzentration von $50 \mathrm{~g} / \mathrm{l}$ ); geprüft wurden der Einfluß $\beta$ von steigenden Mengen Fibrinogen. $N=6$ Proben pro gleichbleibender Fibrinogenmenge (Median, Minimum, Maximum).

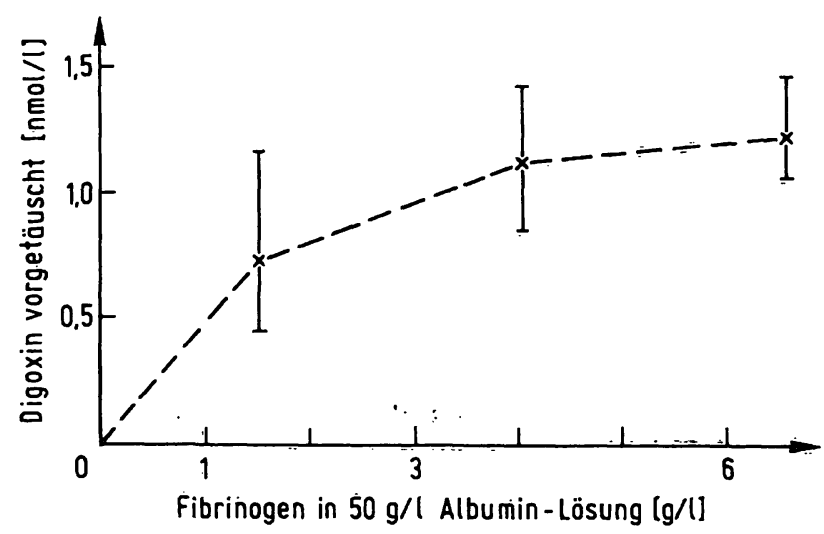

Abb. 7. Vortäuschung voñ Digoxin im digoxinfreien Milieu durch Fibrinogen; Matrix der Probe war eine Albuminlösung mit einer Proteinkonzentration von 50 $\mathrm{g} / \mathrm{l}$; geprüft wurde der Einfluß von steigenden Mengen Fibrinogen. $\mathrm{N}=12$ Proben pro gleichbleibendem Fibrinogengehalt (Median, Minimum, Maximum).

Nach Recalcifizierung von Citratplasma und an: schließender Abtrennung des Fibrins entsprechen die Digoxinmengen, die zuivor im Plasma zu hoch gemessen wurden, dann weitgehend den Serumwerten. Un= terschiede nach dem $\mathrm{t}$-Test für Paar-Analysen lassèn sich nicht mehr sichern (Abb. 8).

Wird Plasma $30 \mathrm{~min}$ lang auf $56^{\circ} \mathrm{C}$ erhitzt, denaturiert Fibrinogen und kann, unlöslich geworden, durch -Zentrifugation abgetrennt werden (5). Da Digoxin unter diesen Bedingungen staḅil ist (Tab. 2), kann 


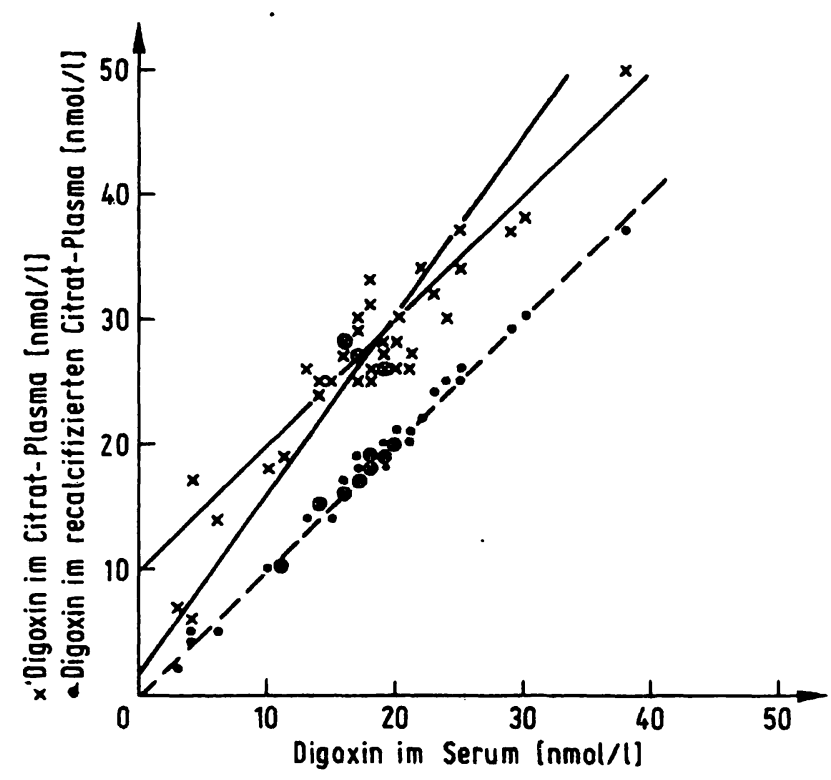

Abb. 8. Korrelation zwischen Digoxin-Werten im Serum und im Plasma $(x)$ sowie nach erfolgter Defibrinierung des Plasmas durch Recalzifizierung (•):

für Plasma:

$N=40 ; x=-0,124+0,68 \cdot y ; y=1,07+0,97 \cdot x$;

$r=0,8104 ; t=8,53 ; p<0,001$

$\mathrm{t}$-Test für Paar-Analysen:

$t=13,14 ; p<0,001$

für durch Recalzifizierung defibriniertes Plasma:

$\mathrm{N}=40 ; \mathrm{x}=0,010+0,988 \cdot \mathrm{y} ; \mathrm{y}=0,012+0,9996 \cdot \mathrm{x}$;

$r=0,9938 ; t=54,92 ; p<0,001$

$t$-Test für Paar-Analysen:

$\mathrm{t}=1,11 ; 0,25<\mathrm{p}<0,3$

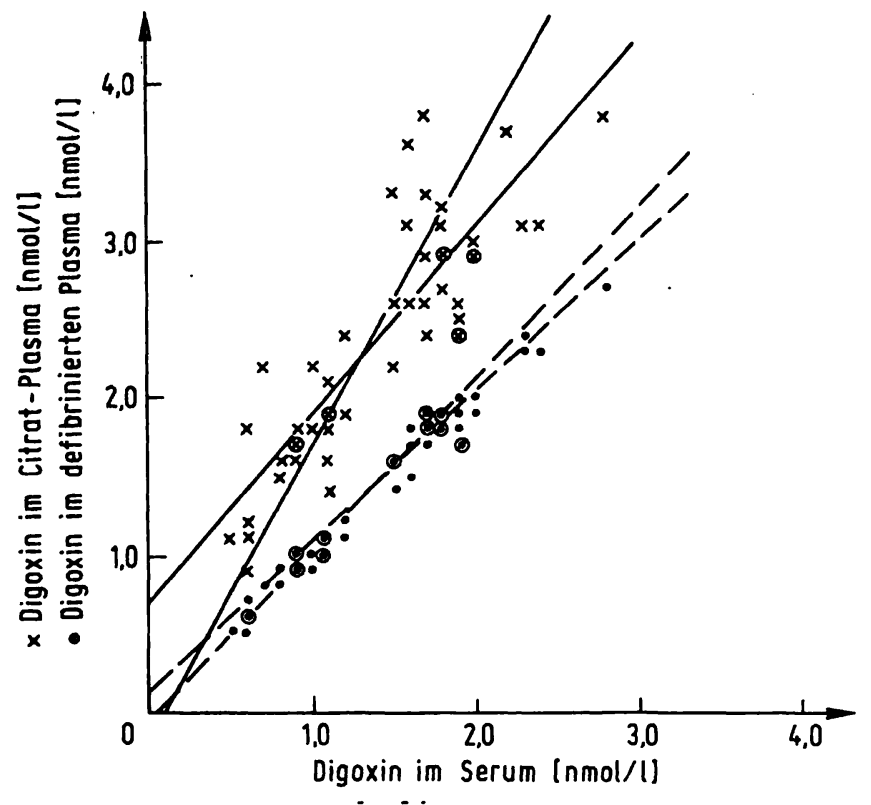

Abb. 9. Korrelation zwischen Digoxin-Werten im Serum und im Plasma $(x)$ und nach erfolgter Defibrinierung des Plasmas durch thermische Denaturierung des Fibrinogens (•):

für Plasma:

$\mathrm{N}=50 ; \mathrm{x}=0,083+0,552 \cdot \mathrm{y} ; \mathrm{y}=0,707+1,20 \cdot x$;

$r=0,8123 ; t=9,65 ; p<0,001$

$\mathrm{t}$-Test für Paar-Analysen:

$t=16,82 ; p<0,001$

für durch thermische Denaturierung defibriniertes Plasma:

$N=50 ; x=0,028+0,936 \cdot y ; y=0,146+0,942 \cdot x$

$r=0,939 ; \mathrm{t}=18,92 ; \mathrm{p}<0,001$

t-Test für Paar-Analysen:

$\mathrm{t}=1,388 ; 0,1<\mathrm{p}<0,2$

Die Beseitigung des Fibrinogens aus der Probe erweitert das jeweilige Analysenverfahren durch zusätzliche Arbeitsschritte. Bessere Praktikabilität der Handhabung des Testes böten Zusätze zur Immunreaktion, durch die die fibrinogenbedingte Störung sich beseitigen ließe.

Durch Harnstoffzusatz zum Milieu der Immunreaktion im Enzymun-Test ${ }^{\circledR}$ Digoxin besteht die Möglichkeit, den störenden Effekt des Fibrinogens zu beeinflussen. Als wirkungsvoll erwiesen sich am Beispiel einer Plasmaprobe, die mit 1,7 nmol/l Digoxin aufgestockt wurde, Modifikationen mit einem Zusatz von $0,3 \mathrm{~mol} / 1 \mathrm{Harnstoff}$ zur Inkubationslösung der Antigen-Antikörperreaktion und einer Inkubationsdauer für diese Reaktion von 2,5 Stunden. Unter diesen Testbedingungen betrug die Wiederfindung der zugesetzten Digoxinmengen zum Plasma $100 \%$, die bei Verwendung der Originalreagenzien mit $235 \%$ falsch zu hoch angezeigt wurde (Abb. 10a, b). Die beschriebenen Bedingungen reichen aber nicht aus, den störenden Fibrinogeneffekt in jeder beliebigen Plasma- 

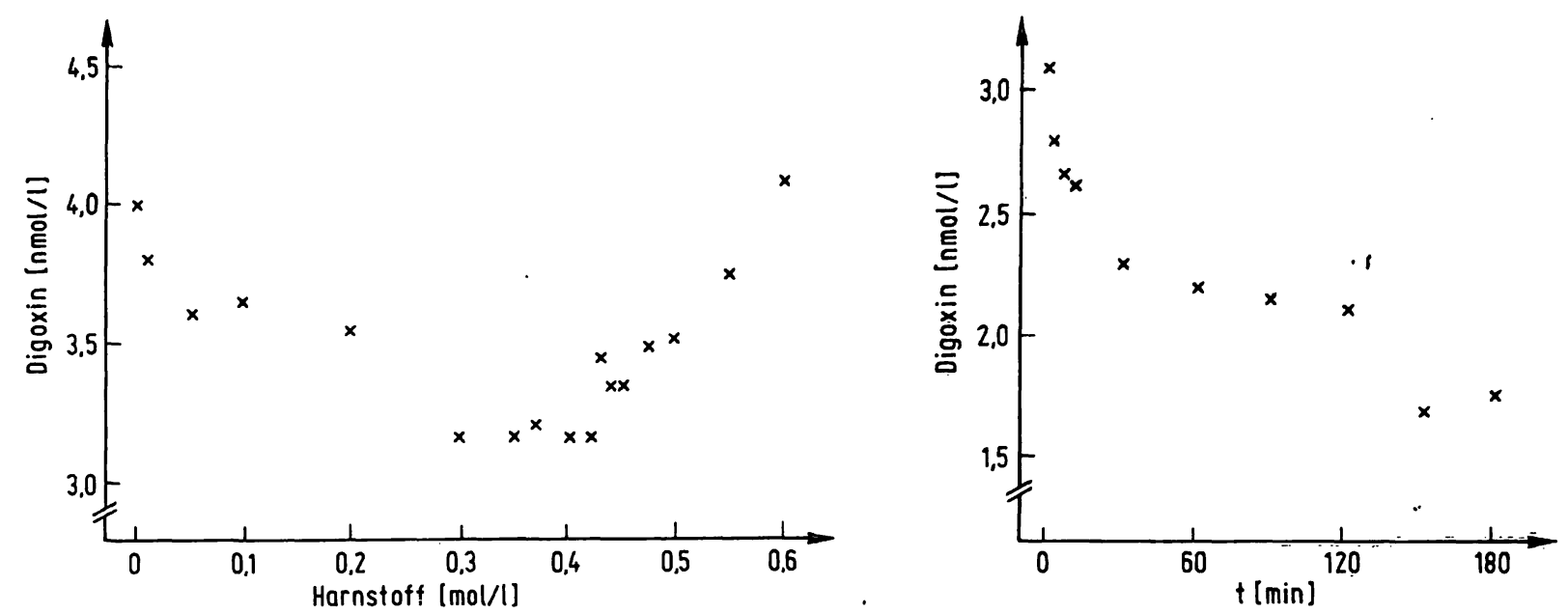

Abb. 10. Veränderungen der Inkubationsbedingungen der Antigen-Antikörper-Reaktion im Enzymun-Test ${ }^{\text {Ti }}$ Digoxin.

a) Harnstoffzusatz

b) Verlängerung der Inkubationszeit und Zusatz von $0,3 \mathrm{~mol} / 1$ Harnstoff zur Inkubationslösung.

Soll-Wert des Plasmas $=1,7 \mathrm{nmol} / \mathrm{l}$ Digoxin.

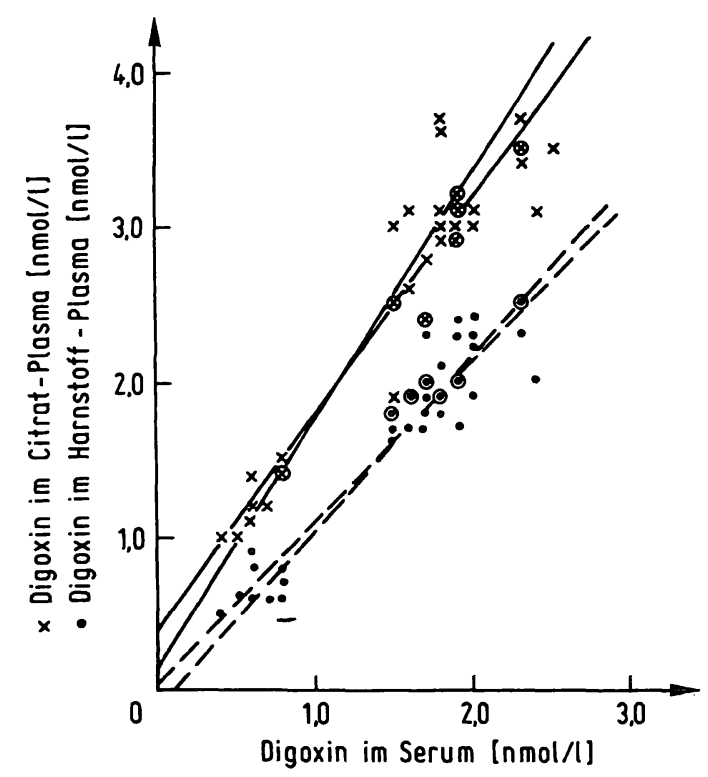

Abb. 11. Korrelation zwischen Digoxin-Werten im Serum und im Plasma (x) nach Zusatz von $0,3 \mathrm{~mol} / 1$ Harnstoff zur Inkubationslösung der Immunreaktion und Verlängerung der Inkubationsdauer auf insgesamt 2,5 Stunden (๑):

für Plasma:

$\mathrm{N}=40 ; \mathrm{x}=-0,117+0,644 \cdot \mathrm{y}$

$y=0,460+1,378 \cdot x$

$r=0,9424 ; \mathrm{t}=17,30 ; \mathrm{p}<0,001$

$\mathrm{t}$-Test für Paar-Analysen:

$\mathrm{t}=18,12 ; \mathrm{p}<0,001$

für Zusatz von $0,3 \mathrm{~mol} / 1 \mathrm{Harnstoff}$ und $2,5 \mathrm{~h}$ Inkubation:

$\mathrm{N}=40 ; \mathrm{x}=0,088+0,877 \cdot \mathrm{y} ; \mathrm{y}=0,059+1,043 \cdot \mathrm{x}$

$r=0,9561 ; t=20,12 ; p<0,001$

$\mathrm{t}$-Test für Paar-Analysen:

$\mathrm{t}=-4,31 ; \mathrm{p}<0,001$ probe vollständig zu beseitigen. Entsprechend unbefriedigend sind die in Abbildung 11 dargestellten Ergebnisse.

Diese lassen den Schluß zu, daß Harnstoffzusatz und Inkubationszeit probenabhängig sind. Das Beispiel der Abbildung 10a zeigt darüber hinaus deutlich, da $B$ die Harnstoffwirkung limitiert ist. Harnstoffmengen über $0,3-0,4 \mathrm{~mol} / 1$ in der Inkubationslösung bewirken wieder einen ungünstigen Effekt im Sinne einer weiteren Vortäuschung von Digoxin. Dieses Phänomen beruht vermutlich auf einem zusätzlichen Verlust wandständiger Antikörper durch Harnstoff.

\section{Diskussion}

Die in den Abbildungen 5, 6,7 dargestellten Untersuchungsergebnisse zeigen, daß Fibrinogen als Matrixbestandteil der Probe im heterogenen enzymimmunologischen Test einen Einfluß auf das Testergebnis hat. Soweit der Test auf dem Kompetitionsprinzip beruht, täuschen steigende Mengen Fibrinogen steigende Mengen der nachzuweisenden Substanz vor (Abb. 6, 7). Demgègenüber führen im Sandwich-Prinzip, z. B. im Enzymun-Test ${ }^{\circledR}$ AFP (Abb. 5), steigende Mengen Fibrinogen in der Probe zu einer entsprechenden Abnahme der meßbaren Substanzmengen. Enthielten die Modellproben für diesen Test kein $\alpha_{1}$-Fetoprotein, hatte der Fibrinogengehalt der Probe keinen Einfluß auf die Meßergebnisse. Diese Un̦țersuchungen lassen 
den Schluß zu, daß Fibrinogen als kreuzreagierendes Antigen nicht in Betracht kommt. Das Fibrinogen bewirkt offenbar einen Verlust wandständiger Antikörper, da es als oberflächenwirksame Substanz die mit Antikörpern beschichtete Innenseite des Reaktionsgefäßes zu überziehen vermag. Die Folge ist eine sterische Behinderung der für die Immunreaktion in Frage kommenden Molekülabschnitte. Betroffen werden von diesem Vorgang vor allem solche Antikörpermoleküle, deren reaktionsfähiger Molekülbereich zur Gefäßwand den geringsten Niveauunterschied hat. Der mengenmäßige Anteil so postierter Antikörper an der Gesamtmenge wandständiger Antikörper wird in besonderem Maße einen Einfluß auf die Präzision der Immunreaktion haben und den Fibrinogeneffekt zusätzlich beeinflussen.

Bei Vergleichsuntersuchungen mit Plasma und Serum werden mit dem Enzym-Test ${ }^{\oplus}$ AFP, Enzymun-Test ${ }^{\oplus}$ Digoxin und Enzygnost ${ }^{\circledR}$ PAP Unterschiede in den jeweils gemessenen Mengen gefunden, die nach dem t-Test für Paar-Analysen statistisch gesichert sind (Abb. 1, 3, 4). Mit dem Cortisol-sopheia ${ }^{\mathrm{TM}}$-Test lassen sich zwischen Plasma und Serum Unterschiede in den gemessenen Cortisolmengen nicht sichern (Abb. 2). Das Antikoagulans ergibt bei allen Prüfungen keine zusätzlichen Störungen.

Besonders bei den Vergleichsuntersuchungen der Bestimmung von saurer Prostata-Phosphatase (Abb. 4a, $b, c)$ fällt auf, $\mathrm{da} ß$ bei einzelnen Paaruntersuchungen erhebliche Unterschiede bestehen. Sie werden im Rahmen der Versuchsanordnung häufiger beobachtet und als Ausdruck einer ungünstigen Qualität in der Wandbeschichtung der Röhrchen mit Antikörpern und einer daraus resultierenden begrenzten Bindungsfähigkeit gedeuteț. Es wäre denkbar, daß Fibrinogen diesen Effekt zusätzlich verstärkt.

Der Test Cortisol-sopheia ${ }^{\mathrm{TM}}$ liefert weitgehend übereinstimmende Ergebnisse aus Plasma und Serum (Abb. 2a, b, c). Die durch Fibrinogen in diesem Test vorgetäuschten Cortisolmengen sind gering (Abb. 6). Enthält das Plasma physiologische Fibrinogenmengen und auch Cortisolmengen im Referenzbereich, bleibt im Plasma der durch Fibrinogen vorgetäuschte Cortisolanteil unter 5\% (vergl. Abb. 2 und 6), der für dạs Ergebnis im Plasma nicht rẹe lẹant wird.

Die gefundenen Mengenunterschiede für $\alpha_{1}$-F̂etoprotein zwischen Plasma und Serum sind mit dem angewandten Verfahren gering. Nach persönlichen Informationen enthält das Reaktionsmilieu für die Immunreaktion in diesem Test einen Zusatz, der eine fibrinogenbedingte Störung beeinflußt. Der zugesetzte Stoff ist aus patentrechtlichen Gründen unbekannt.
Die bei Einsatz von Plasma als Probenmaterial bestehende Problematik läßt sich vermeiden, wenn Fibrinogen aus der Plasmaprobe entfernt wird oder durch geeignete Zusätze zur Immunreaktion der vom Fibrinogen ausgehende Störeffekt beseitigt wird. Am Beispiel der Digoxinbestimmung mit dem EnzymunTest ${ }^{\oplus}$ Digoxin sollen geeignete Möglichkeiten hierzu diskutiert werden.

Ohne Einfluß auf den EIA ist die vorangehende Entfernung von Fibrinogen aus der Plasmaprobe durch Recalcifizierung. Damit wird die Gerinnungskaskade gestartet, das entstandene Fibrin läßt sich anschlieBend abzentrifugieren. Die Digoxinwerte aus so vorbehandelten Plasmaproben entsprechen dann den Serumwerten (Abb. 8).

Digoxin erwies sich bei $56^{\circ} \mathrm{C}$ als thermostabil (Tab. 2). Wird Plasma bei $56^{\circ} \mathrm{C} 30 \mathrm{~min}$ lang erhitzt, wird eine thermische Denaturierung von Fibrinogen in der Probe erreicht. Fibrinogen wird unlöslich, kann durch Zentrifugation abgetrennt werden. Die gemessenen Digoxinmengen im Serum und im nach dem beschriebenen Verfahren defibrinierten Plasma sind nach dem t-Test für Paar-Analysen vergleichbar (Abb. 9).

Durch die Einführung eines zusätzlichen analytischen Schrittes zur Beseitigung von Fibrinogen wird der Test arbeitsintensiver. Deshalb wären geeignete Zusätze zur Immunreaktion mit der Möglichkeit, den Fibrinogeneffekt zu beeinflussen, eine elegantere Lösung. Die sich hierbei ergebende Problematik wird am Beispiel der Digoxinmessung mit dem EnzymunTest ${ }^{\oplus}$ Digoxin aufgezeigt. Als Zusatz wurde Harnstoff gewählt. Harnstoff vermag bis zu einer bestimmten Menge im immunologischen Reaktionsmilieu den Fibrinogeneffekt $\mathrm{zu}$ beeinflussen. Die Harnstoffwirkung ist aber dadurch limitiert, daß Überschreitungen einer bestimmten Menge dann ebenfalls wieder zur Digoxinvortäuschung führen. Offenbar bewirken größere Harnstoffmengen eine zusätzliche Ablösung wandständiger Antikörper. Der Harnstoffeffekt kann aber durch die Inkubationszeit optimiert werden (Abb. 10b). Mit der beschriebenen Modifikation der Immunreaktion wird an einem Beispiel einer Plasmaprobe, die mit einer definierten Menge Digoxin aufgestockt wurde, gezeigt, daß der Sollwert für Digoxin wiedergefunden werden kann (Abb.10a, b). Diese Testbedingungen lassen sich aber offenbar nicht auf jede individuelle Plasmaprobe anwenden. Trotz der Anwendung der für Plasmaproben beschriebenen Modifikation durch Harnstoff sind an 40 Paaruntersuchungen die Meßwerte für Serum und Plasma nach dem t-Test für Paar-Analysen weiterhin signifikant verschieden. Ein geringer Harnstoffeffekt ist bei diesen aufgeführten Plasmaproben allerdings erkennbar. 
Es wird angenommen, daß der für eine probenindividuelle Beseitigung des Fibrinogeneffekts benötigte Harnstoffzusatz von der in der Probe enthaltenen Fibrinogenmenge abhängig ist (Abb.11).

\section{Literatur}

1. Colombo, J.P. (1978) in Klinische Chemie, Theorie, Praxis, Interpretation (Richterich, R. \& Colombo, J.P., eds.) 4. Auflage pp. 84-87, Verlag S. Karger, Paris, London, New York, Sydney.
Wenn die Erfahrungen aus den vorliegenden Beispielen gedeutet werden, wird aus Gründen erreichbarer Richtigkeit dèr Testergebnisse der Einsatz von Serum als Probenmaterial dem Plạsma vorzuziehen sein.

2. Allner, R. \& Krüpe, H. (1981) Ärztl. Lab. 27, 69-73.

3. Clauss, A. (1957) Acta Haematol. 17, 237-243.

4. Weichselbaum, T. E. (1946) Amer. J. Clin. Pathol. 10, 40-49.

5. Schulz, F. H. (1955) Acta Hepatologica 3, 306-309.

Priv. Doz. Dr. med. Rolf Allner Chefarzt Zentrallabor Städt. Kliniken Pacelliallee 4

D-6400 Fulda 